\title{
PROPERTIES AND DISTRIBUTION OF GALAXIES \\ IN RELATION TO THEIR FORMATION AND \\ EVOLUTION: THEORETICAL SITUATION
}

\author{
P. J. E. PEEBLES \\ Dept. of Physics, University of California, Berkeley, Calif., and \\ Joseph Henry Laboratories, Princeton University, Princeton, N.J., U.S.A.
}

\begin{abstract}
This report is a review of several proposed pictures for how matter came to be concentrated in lumps like galaxies and clusters of galaxies. The proposals cover a broad range of unrelated possibilities. I attempt here to subject these proposals to critical discussion, to discover the major problems for each case and the more promising lines for further study.
\end{abstract}

\section{Introduction}

The search for some theoretical handle on the significance of galaxies in cosmology centres on three questions:

(1) According to accepted or conjectured laws of physics, what are the processes by which structure on the scale of galaxies can form and evolve? This has been the subject of several recent reviews, ${ }^{*}$ and for the most part I will not attempt to repeat any of the computational details here.

(2) What are the phenomena that bear clearest witness to processes of evolution? This question seems fair enough, but we recognize that it is loaded. The great trick is to pick out from the welter of peripheral effects those phenomena that have a direct bearing on the central issues of origin and evolution. The great danger is that our decisions about what is direct and what is peripheral depend on our prejudices about what is the best candidate for the physical process.

(3) Which of the processes listed under question (1) fit into the astrophysical situation, as delineated under (2), without logical contradiction or undue forcing? Again, we should approach this question with some care. One can find in the literature many different proposals for how galaxies may have formed, and in many cases the author shows how, if all works out as conjectured, the picture will account for a broad variety of phenomena. This is hardly surprising. There is a strong systematic bias against publishing models that do not give the 'right' answers. Also, by the nature of the problem, the models are often so complicated that it is hard to make definite statements about how they really would behave. One must introduce conjectures, and it is a natural human tendency to choose the optimistic possibility at each step along

* For different aspects of the problem see the preprint by G. B. Field (intended for the fabled Volume 9 of Stars and Stellar Systems), the review papers in Supplement 49 of Progress of Theoretical Physics (1971), the review by Rees (1971), and the textbooks Physical Cosmology (Peebles 1971a; hereafter referred to as PC), Chapter 7, and Gravitation and Cosmology (Weinberg, 1972; hereafter called GC), Chapters 8-10. 
the way. The result is that the 'big picture' in this subject is uncertain ground indeed. Under the circumstance it may be best to narrow the line of attack, to seek out the spots of firmer ground here and there where some aspect of the situation appears clean and simple, and we might see how one or another of the proposed schemes may be put to serious and objective test. It would be unrealistic to hope that this approach will soon lay to rest any of the more popular schemes for galaxy formation, but it should show the way to less cluttered ground for the debate.

In thinking about how to attack this third question, we may find it helpful to recall the role of uniformitarianism in the origins of modern geology (in the 18th century). One argued that observed geologic features might be understood as the result of observed processes (like erosion and sedimentation) acting over a sufficiently long time. A most beneficial feature of this concept was (and is) that it allowed people to approach the problem in a rational and scientific way without having to address the enormously complicated ultimate issue of the origin of the Earth. I suspect that our attempts to find a theoretical account of galaxies in cosmology has been unduly influenced by the fact that modern 'establishment cosmology' came into being in a peculiar way, through the inspired guesses of a few people. Even if these guesses prove accurate we have no reason to think that we can repeat the coup. But even if we lack inspired guesses we can fall back on the uniformitarian method. I would state the lesson as follows: (1) form an opinion of the relevant 'observed' phenomena; (2) draw up a list of presumed processes and/or laws of physics; (3) ask whether one can trace back in time from the phenomena according to the processes without running into a situation that appears contrived or contradictory.

Of course this is a slow and uncertain business. The singular big-bang surely does violence to uniformitarianism - so do we blame the assumed laws of physics - general relativity in this instance; the assumed situation - a uniformly expanding Universe; or the notion of uniformitarianism? Still, it offers a clear line of attack, which is to be treasured.

In the present review I consider mainly structure on scales larger than galaxies groups, clusters and superclusters. This is somewhat out of line with the main subject of the conference, the nature of galaxies, but the phenomenon surely is closely related, and the great attraction is that the physics appear to be a good deal simpler. We might even hope that this large-scale structure is a reasonably undisturbed fossil of what was happening in the past, and at the very least the apparent simplicity of the phenomenon strongly limits the theoretical speculation. In contrast, the galaxies seem like fossils that have been pulverized by the complex processes of evolution.

Not included in this review is the search for young galaxies - perhaps newly-formed objects in our neighbourhood of the Universe, perhaps objects at high redshift. Its importance can hardly be overstated, however. Once we have a genuine young galaxy in our hands, so to speak, it will settle a lot of arguments.

I base the discussion on the standard big-bang cosmological model. This is an assumption, and not one that all would adopt, for the big-bang cosmology is not established beyond reasonable doubt. On the other hand, my impression of the observa- 
tional situation is that this cosmology has strong credentials as a working hypothesis.* In an effort at ecumenicity I have sought where possible to frame arguments that are more broadly based than the big-bang picture. I have also restricted the discussion to redshifts $z \lesssim 1000$. This eliminates an active field of research, the possible role of the primeval fireball radiation in shaping and controlling the evolution of irregularities. This subject may prove to be of decisive importance to the problem of galaxy formation, but it is still quite complex and highly uncertain.

\section{Large-Scale Irregularities in the Matter Distribution}

The nature of the large-scale distribution of the observed matter, as galaxies, has been reviewed by de Vaucouleurs (1971). At this conference G. Burbidge (p. 93) has discussed a second component of possibly decisive importance, the intergalactic phenomena. I only wish to describe here a particular aspect of the observational situation that seems to me to be of considerable interest - the clouds of galaxies in the Shane-Wirtanen Catalog (1967) and the superclusters found in the distribution of rich clusters cataloged by Abell (1958). Shane and Wirtanen counted galaxies down to limiting brightness $m_{\mathrm{pg}} \lesssim 19$, which means that the galaxies they counted are typically at distances in the range of $200 h^{-1} \mathrm{Mpc}$ to $400 \mathrm{~h}^{-1} \mathrm{Mpc}{ }^{* *}$ The published data give the counts in $1^{\circ} \times 1^{\circ}$ cells. These counts are correlated over angular distances as large as $5^{\circ}$, which corresponds to a spatial coherence or correlation in the galaxy distribution over distances as large as $\sim 30 \mathrm{~h}^{-1} \mathrm{Mpc} . \ddagger$ Following Zwicky and Shane, this phenomenon is conveniently described as the 'cloudy' nature of the large-scale distribution of galaxies. The clouds are considerably bigger than the rich clusters cataloged by Abell, but we know that the rich clusters must be in the clouds of galaxies because the Shane-Wirtanen galaxy counts show a strong cross-correlation (over angular scales $\lesssim 5^{\circ}$, depending on cluster distance class) with the positions of the Abell cluster centres (Peebles, 1974). What is more, there is convincing evidence of correlations amongst the positions of the Abell clusters. ${ }^{\dagger}$ The observed correlation

\footnotetext{
* For a less optimistic view see Burbidge (1971). For a recent review of the current observational situation in cosmology see PC. The only significant change since PC is the status of the microwave background shortward of $1 \mathrm{~mm}$ wavelength. All observers now appear to be agreed that there is no evidence for or against a black-body spectrum shortward of $1 \mathrm{~mm}$ wavelength (Houck et al., 1972; Williamson et al., 1973; Muehlner and Weiss, 1973).

** All lengths, times, etc. are based on a nominal value for Hubble's constant, $H=100 \mathrm{~km} \mathrm{~s}^{-1} \mathrm{Mpc}^{-1}$, $H^{-1} \cong 1 \times 10^{10} \mathrm{yr}$. The dimensionless number $h$ is the 'true' value of Hubble's constant measured in units of $100 \mathrm{~km} \mathrm{~s}^{-1} \mathrm{Mpc}^{-1}$. This very convenient practice is becoming common, but it was too much to hope that people would settle on a standard nominal value - in different papers one can find the same symbol $h$ used to represent Hubble's constant in units of 50,75 , and $100 \mathrm{~km} \mathrm{~s}^{-1} \mathrm{Mpc}^{-1}$.

* The Lick counts were analyzed by Limber (1954) and by Neyman et al. (1953). For a recent detailed rediscussion see Peebles and Hauser (1974).

+ The first demonstration of superclusters that I found truly clean and convincing was that of Bogart and Wagoner (1973). Hauser and I had independently attacked the problem, following the method used earlier to set an upper limit on superclustering (Yu and Peebles, 1969), and we independently concluded that superclusters do indeed exist, at about the earlier upper limit. The numbers quoted here are from Hauser and Peebles (1973).
} 
has roughly the same linear scale as that of the clouds, and there are about two clusters per supercluster, in the sense that there is on the average $\sim 1$ cluster in excess of random near a randomly chosen cluster. The superclustering or correlations among cluster centres again extends to lengths $\sim 30 \mathrm{~h}^{-1} \mathrm{Mpc}$.

The question of structure on scales larger than 20 to $30 \mathrm{~h}^{-1} \mathrm{Mpc}$ has been reviewed by de Vaucouleurs (1971). The observational problems are very difficult, so I have chosen not to consider such effects here. As for the clouds (superclusters), many details of the galaxy organization and motion remain obscure. However, the simple fact that we can detect structure on scales as large as $30 h^{-1} \mathrm{Mpc}$ gives some interesting constraints on models for evolution, as is described below.

\section{The Evolution of Irregularities}

\subsection{White HOLES}

Phenomena associated with galactic nuclei have occasioned in a number of different contexts the thought that galaxies might have issued out of primeval nuclei or 'white holes.' $*$ This is a spectacular conception, as befits spectacular and mysterious phenomena, and for that very reason it seems clear that its role will be understood only when we have a much clearer understanding of the physics of galaxies. We can observe, however, that if each galaxy issues out of its own primeval nucleus then we are left with the problem of accounting for the large-scale clustering of primeval nuclei, in the clusters and clouds of galaxies. It has been suggested that only the dominant galaxy in a group or cluster is associated with a primeval nucleus, the lesser members having formed by some sort of calving process (e.g. Hoyle, 1965, pp. 19, 20). But how does one account for the clouds of galaxies? Here is a true hierarchical structure, groups and clusters within clouds. In the present scheme, we must assume either that the primeval nuclei are distributed in a hierarchical fashion, or else that the primeval nuclei occur one to a cloud, the ejected matter fragmenting down the hierarchy into clusters, groups and galaxies. Either answer seems to lead us back to the original problem.

A related difficulty is that of the peculiar velocities of galaxies. If the galaxies in a cloud issued from one source, then to spread over $30 h^{-1} \mathrm{Mpc}$ in one expansion time $\sim 10^{10} h^{-1} \mathrm{yr}$, the matter would have to have a dispersal velocity $\sim 3000 \mathrm{~km} \mathrm{~s}^{-1}$. If the dispersal velocity were close to this limit the expansion of the cloud would roughly mock the general expansion of the Universe in the big-bang cosmology. If the dispersal velocity were much less, the galaxies would have appeared tightly

* Early references to this concept are found in Jeans (1928) and Milne (1948). In cosmological discussions, McCrea (1964) considered 'embryos' of galaxies ejected from old galaxies, and Hoyle and Narlikar (1966) considered 'pockets of creation' of matter. The subject was first vigorously pursued from the point of view of phenomenology by Ambartsumian $(1958,1965)$. Other studies of phenomena that may point to (or test) the concept are described by Arp at this conference (p. 199, cf. also Arp 1971 and earlier references therein), Burbidge et al. (1963), Hoyle (1965), Holmberg (1969), Bahcall and Joss (1972) and van der Kruit et al. (1972). Novikov (1964) and Ne'eman (1965) discussed a similar picture for the nature of quasars. 
concentrated around the sources, which is not observed. If the velocity were much greater, say $6000 \mathrm{~km} \mathrm{~s}^{-1}$, twice the critical value, galaxies should now have peculiar velocities on the order of $3000 \mathrm{~km} \mathrm{~s}^{-1}$. This may well be possible for the occasional object, but it is quite unreasonable for the general field. This picture thus requires a very special ejection velocity, which would seem to require some explanation.

These arguments have no direct bearing on the role of primeval nuclei in internal dynamics of galaxies. They may suggest that something else is required to account for the long-range ordering of galaxies.

\subsection{THERMAL INSTABILITY}

The whole panoply of fluid dynamics - thermal phenomena, shocks, turbulence, magnetohydrodynamic effects - may be expected to have played a role in galaxy formation, and to have added to the complexity of these systems. The situation appears much easier for the physics of the cloud structure. This and the following sections deal with several such effects by which people have proposed that irregularities on the scale of galaxies might have evolved. I begin with thermal instability.*

One could suppose that thermal instability acts as the primary agent causing matter which was initially more uniformly distributed to collect in structures like galaxies and groups and clouds of galaxies, or it might be a secondary agent that helps fashion evolution once proto-systems have formed. We are interested here in the former role. This role can be significant if two order-of-magnitude conditions are satisfied - that the velocity of sound in the pre-galactic medium is large enough that a pressure wave can traverse the desired length scale in an expansion time $H^{-1}$, and that the cooling time for the medium is less than or comparable to the expansion time. The first condition applied in the present state of the universe implies that the velocity of sound is $\gtrsim 3000 \mathrm{~km} \mathrm{~s}^{-1}$, or that the matter temperature $\gtrsim 10^{9} \mathrm{~K}$, in order to make irregularities on the scale of $30 h^{-1} \mathrm{Mpc}$. But if the gas is this hot and at the mean cosmological density (or, what is equivalent for this point, at the mean density of the matter within $\sim 300 h^{-1} \mathrm{Mpc}$ ), the cooling time is at least three orders of magnitude larger than the expansion time. It does not help to imagine that the thermal instability occurred in the early Universe, at high redshift, for although the matter density is higher at larger redshift, so is the matter temperature needed to get irregularities on a scale of $\sim 30 h^{-1} \mathrm{Mpc}$ now, the temperature varying as $(1+z)$ in the Einsteinde Sitter model. The bremsstrahlung radiation from this dense relativistic plasma would make for severe problems with the X-ray background. Thus, while it may be that the thermal instability effect played an important role in the evolution of structure on a sub-galactic or even galactic scale, it could not have been a significant factor in the physics of clouds of galaxies.

\subsection{MAGNETOHYDRODYNAMICS}

The origin of the interstellar magnetic field is obscure. One possibility is that the

* Thermal instability in cosmology is discussed by Hoyle (1958), Gold and Hoyle (1959), Field (1965), Kondo et al. (1971) and Arons (1972). 
field existed before the Galaxy. If so, it would be natural to ask whether the magnetic field played a role in the formation of the Galaxy and, by extension, the formation of systems of galaxies.* The dynamic effect of the field on the expanding matter distribution is important if the Alfvén velocity is comparable to the size of the structure of interest divided by the expansion time-scale. For the present values of expansion time-scale, mean mass density, and the size of clouds of galaxies, this criterion yields**

$$
B \gtrsim 5 \times 10^{-6} \Omega^{1 / 2} \text { h G. }
$$

In the interstellar medium the field is $\sim 10^{-6} \mathrm{G}$, but the matter density is some 5 or 6 orders of magnitude higher than the cosmological mean. If the interstellar medium were isotropically expanded down to the cosmological mean density, conserving flux, it would reduce the interstellar field to $\sim 10^{-10} \mathrm{G}$, some four orders of magnitude below the critical value. It might be noted that this factor $10^{4}$ is nearly independent of time in the expanding Universe. $\ddagger$ Thus it appears that, unless the Galaxy is composed of matter which contained usually small flux, magnetic field acting along could not have played a significant role in the behaviour of structure on the scale of clouds of galaxies.

A more interesting question is the possible role of magnetic stresses acting in concert with the gravitational instability effect discussed in Section 3.5 below. A primeval magnetic field would make an anisotropic (and presumably inhomogeneous) contribution to the stress-energy tensor. We might expect to find that, as we trace the expansion of the Universe back in time toward the singularity, this anisotropic stress forces deviations from a homogeneous isotropic expansion (Thorne, 1967). Turning the question around, does this mean that the assumption of a primeval magnetic field would require the assumption of highly special initial conditions to assure that the Universe ends up looking no more irregular than it does? That is, does the assumption of a primeval magnetic field agree with uniformitarianism?

\subsection{Primeval turbulence}

The picture of cosmic turbulence has figured in one form or another in many different

\footnotetext{
* The possible role of magnetic fields in galaxy formation has not received the detailed attention it perhaps deserves. For some discussion see Zel'dovich (1969), Rees (1971), Harrison (1970a), Peebles (1969a), and Rees and Reinhardt (1972).

** $\Omega$ is the ratio of the mean mass density of the Universe (or of the part within a few hundred Mpc distance) to the density in the Einstein-de Sitter cosmological model. Thus the matter density is $Q=2 \times 10^{-29} \Omega \mathrm{h}^{2} \mathrm{~g} \mathrm{~cm}^{-3}$. If the galaxies are the main contribution to $Q$ and if galaxy masses are computed from observed angular sizes and velocity dispersions of galaxies then $\Omega$ is independent of $h$. * The diameter of a chosen system expanding with the Universe varies in proportion to $(1+z)^{-1}$, where $z$ is the redshift. In the Einstein-de Sitter cosmology, $q_{0}=\frac{1}{2}$, the expansion time varies as $(1+z)^{-3 / 2}$, so the critical velocity varies as $(1+z)^{1 / 2}$. Since the Alfvén speed $B \propto Q^{-1 / 2}$, and $Q \propto(1+z)^{3}$, the critical field is $B \propto(1+z)^{2}$, which is also the law for the time variation of $B$ under uniform isotropic expansion and flux conservation. The expansion parameter might be as low as $q_{0} \cong 0.02$. Here, the critical field varies as $B \propto(1+z)^{3 / 2}$ back to $z \cong 50$. At higher redshift the model behaves like the Einstein-de Sitter case.
} 
discussions of galaxy formation. ${ }^{*}$ The attractions are evident. It is a common observation that fluid flow tends to go turbulent - whether it is smoke rising from a cigarette, crude oil flowing in a pipeline, or clouds moving in the interstellar medium. As Gamow (1952) and Oort (1958) have pointed out, the distribution and appearance of galaxies seem like the fossil remnants of turbulent eddies. And for the theorist, turbulence is a lovely point of departure for computation. However, there are some problems.

Let us consider first the possible role of turbulence in the present state of the Universe. We will suppose that matter moves about more or less after the fashion of developed turbulence, in random currents with typical velocity $v_{\mathrm{T}}(\lambda)$ for eddies of size $\lambda$ : These currents can appreciably alter the distribution of matter on the scale of $\lambda$ if $v_{\mathrm{T}}(\lambda) \gtrsim \lambda / t_{0}$, where $t_{0}=H^{-1}$ fixes the expansion time-scale. For the clouds of galaxies this gives the condition, in the present Universe,

$$
v_{\mathrm{T}} \gtrsim 3000 \mathrm{~km} \mathrm{~s}^{-1} \text {. }
$$

But one would expect the peculiar velocities of galaxies and clusters of galaxies to be comparable with the velocities of the large-scale random currents, and it seems that such high peculiar velocities can be ruled out. Of course the situation is much easier if one only wants to make individual galaxies, which would require $v_{\mathrm{T}} \sim 300 \mathrm{~km} \mathrm{~s}^{-1}$. But another problem arises when one attempts to fit these random currents into the big-bang cosmology.

It will be assumed that the pressure of matter and radiation may be neglected compared with $\varrho c^{2}$, where $\varrho$ is the mean mass density. This may be valid back to redshift $z \sim 4 \times 10^{4} \Omega h^{2}$, at which epoch the Primeval Fireball radiation pressure becomes comparable to $\varrho c^{2}$. Now at any epoch the presumed state of turbulence of matter (the matter imagined fairly smoothly distributed in some pre-galactic condition) may be placed in one of two convenient categories, weak turbulence if turbulence velocities $v_{\mathrm{T}}(\lambda)$ on scales $\lambda$ satisfy $v_{\mathrm{T}}(\lambda) t / \lambda<1$, where $t$ is the expansion time-scale, and strong turbulence if the inequality goes the other way. ${ }^{* *}$ As was remarked, if the turbulence is weak nothing much happens in one expansion time because the eddies do not have time to turn over. What is more, one finds that as the Universe expands the number $v_{\mathrm{T}}(\lambda) t / \lambda$, computed for a given eddy mass (that is, computed for a fixed comoving scale of length) decreases: weak turbulence grows.weaker as the Universe expands. Thus, as long as the weakly turbulent eddy expands with the

\footnotetext{
* For a review see Jones and Peebles (1972). For somewhat less pessimistic views of turbulence see for example Oort (1970), Ozernoy (1971), Stecker and Puget (1972).

** For various parts of the argument given here see Peebles (1971b) and Jones (1973). Apparently the process of turbulent dissipation was first discussed in this context by Tomita et al. (1970). An elegant and considerably more rigorous discussion of some aspects of how strong and weak turbulence evolve is given by Olsen and Sachs (1973). Silk and Lea (1973) have discussed the evolution of primordial random motions of galaxies. The problem is similar to that of turbulent dissipation, but since galaxies are compact the collision rate would be lower than for a more nearly uniformly distributed pre-galactic medium, and the direct collision of two galaxies would be less catastrophic than the collision of two eddies in a gaseous fluid.
} 
general expansion of the Universe, the eddy never will have time to turn over, or to alter appreciably the matter distribution. As in the discussion of primeval magnetic fields, we can note that a more interesting situation is obtained when we consider the combined effects of weak turbulence and gravitational instability - the weak matter currents feed growing modes of density irregularity (Peebles, 1971b; Ozernoy, 1971). However, it seems difficult to distinguish this situation from the straight assumption that there are small density irregularities in the early Universe.

If the turbulence is strong, eddies can turn over. According to conventional understanding the eddies in this case are strongly unstable, so that in one eddy turn-over time the energy of each eddy is dumped into eddies of smaller scale and/or shocks, depending on how $v_{\mathrm{T}}$ compares with the velocity of sound. The former effect is a result of inertia, not viscosity, and is thought to lead to the Kolmogorov spectrum for fully developed turbulence. Of course, it may be that the primeval turbulence eddies are stabilized by some unexpected effect, as is enjoyed by tornadoes (cf. the 'spinning cores' concept of Harrison, 1970b). The point is important, for in the absence of some stabilization effect, we must expect that the energy in strong primeval turbulence would rapidly cascade down to smaller and smaller eddies until it is dissipated by viscosity. If so, we are in a bind. If we assume that at some epoch $t$ primeval turbulence was playing a significant role in the evolution of irregularities, then we expect that at epochs earlier than $t$ the turbulence was even stronger and the role even more significant.

There are three ways to avoid the blow-up:

(1) Spontaneous Generation of Turbulence. The general expansion of the Universe is a fluid motion, with which one can associate a Reynolds number that is enormous. It is natural to ask whether this general expansion might break up into turbulent motion. The linear perturbation calculation says that this does not happen if the expansion is nearly homogeneous and isotropic - for only compressional (acoustic) perturbations grow, and these only as a power of time. The situation is more difficult if the expansion is highly anisotropic.* However, the precise isotropy of the microwave background shows that the expansion in fact must have been nearly isotropic at least back to a redshift $z \sim 1000$.

(2) Driven turbulence. Turbulent motion in intergalactic (or pre-galactic) matter might be a secondary phenomenon driven by local disturbances like quasars, young galaxies, regions of matter-antimatter annihilation, or primeval nuclei.** This merits further careful study, for it can neatly avoid the bind and open the way for turbulence phenomena at modest redshift, when galaxies might be expected to have formed.

\footnotetext{
* For conflicting indications on the generation of turbulence in anisotropic cosmological models, see Silk (1973) and Perko et al. (1972).

** Stecker and Puget (1972) discuss turbulence driven by matter-antimatter annihilation. In the picture discussed by Doroshkevich et al. (1967), turbulence (or mass motion sufficient to initiate galaxy cluster formation) is driven by heating by massive protostars. Silk and Solinger (1973) discuss turbulence driven by active radio galaxies. One might include in this category Sciama's (1955) picture of matter motion driven by the motion of galaxies through the intergalactic medium.
} 
(3) Turbulence in the radiation-dominated phase. One considers here turbulence at high redshift, $z \gtrsim 4 \times 10^{4} \Omega \mathrm{h}^{2}$. There are two new effects - the Primeval Fireball radiation would have been coupled to the matter through Thomson scattering, and the mass density of the radiation would have dominated that of the matter. Under these conditions, one finds that the expansion of the Universe causes the number $v_{\mathrm{T}}(\lambda) t / \lambda$ to increase with time (while subsequent to $z \sim 4 \times 10^{4} \Omega \mathrm{h}^{2}$ the expansion of the Universe causes the number to decrease). Thus weak turbulence can evolve into strong turbulence, so one can assume that turbulence is primordial.* The very attractive feature of this picture is that the spectrum of density irregularities may be determined up to only a few adjustable parameters by the theory of fully developed turbulence. The main difficulty is the question of what happens subsequent to the epoch at redshift $z \sim 1000$ when, in the absence of any source of excitation, the matter is expected to recombine and decouple from the radiation. Any peculiar matter velocities left over from the turbulence are liable to initiate formation of bound systems sooner than we want them. There are many uncertainties here, but the dimensions of the problem perhaps are indicated by the following outline of the simpleminded arguments.

The turbulence can be considered to be fully developed (that is, in the regime of of strong turbulence) for some range of eddy sizes. Rough estimates for upper and lower bounds of this range are shown as lines (1) and (2) in Figure 1. The existence of an upper bound $\lambda_{m}$ on the eddy size follows from the assumption, which will be adopted hers, that the turbulence velocities are non-relativistic, $v_{\mathrm{T}}<c$. This assures that the turbulence (at $z \sim 1000$ ) can be considered a small perturbation to a FriedmanLemaître cosmological model (Jones, 1973). But then $\lambda_{m}$ satisfies $\lambda_{m} / t \equiv v_{\mathrm{T}}\left(\lambda_{m}\right)<c$, so $\lambda_{m}$ is smaller than the horizon $c t$. Since there is an upper bound on the peculiar velocity, the kinetic energy (per unit mass) of the turbulence is bounded. Also, the matter currents in eddies larger than $\lambda_{m}$ may be a large reservoir of kinetic energy, but the linear perturbation calculation says that this energy, in weakly turbulent eddies, is not fed to smaller-scale eddies. Now this limited fund of kinetic energy of the developed turbulence is dissipated in two ways. First is the general expansion of the Universe: in effect the expansion adiabatically 'cools' the random currents. Second is the turbulent dissipation process mentioned above, the cascade of energy from large eddies to smaller ones, the energy ending up in eddies small enough to be dissipated by viscosity or whatever. Figure 1 shows the possible course of evolution of $\lambda_{m}$ at epochs near redshift $z=1000$. The vertical axis is a co-moving length scale, that is, the length is adjusted to take account of the general expansion from the epoch $z$ to the present. The right-hand scale is the mean mass (in solar masses) contained within a sphere with diameter equal to the length plotted on the left-hand

* This result was first exploited by Ozernoy and Chernin (1967). For recent discussions see Ozernoy (1971), Silk and Ames (1972), Dallaporta and Lucchin (1972), Jones (1973), Stein (1974). Zel'dovich and Novikov (1970) have remarked that even taking account of the fireball radiation the primeval turbulence picture has the unfortunate property that, when traced back to the singularity in the big-bang model, it gives rise to divergent irregularities in curvature. 


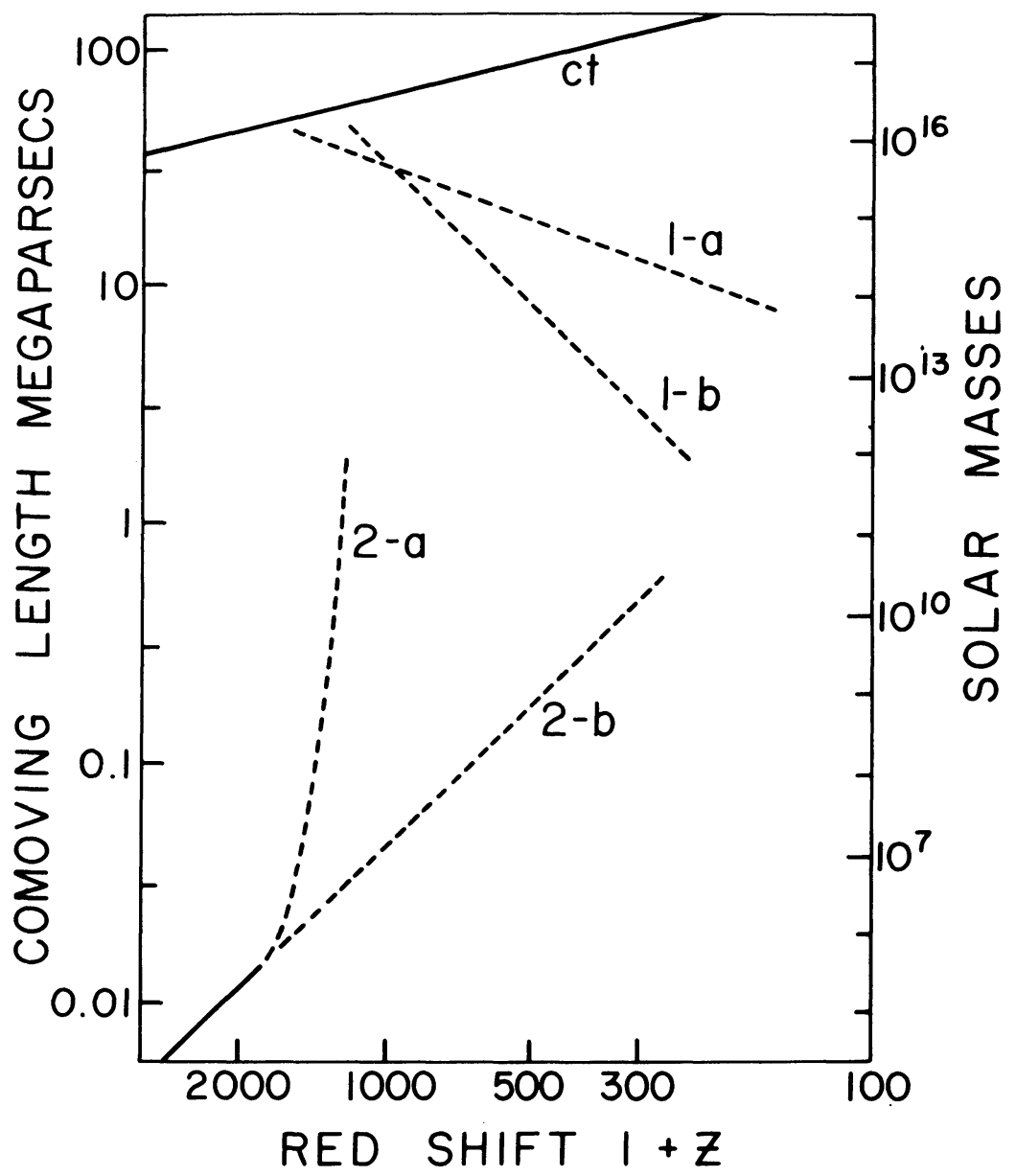

Fig. 1. Limits of primeval turbulence in the early Universe.

scale. In plotting this figure, I have assumed $\Omega=h=1$. Other values for these parameters change the numbers but not the general idea. The bounding wavelength $\lambda_{m}$ has been adjusted to pass through $30 \mathrm{Mpc} /(1+z)$ at $z=1000$. This is about as large as it could be, consistent with the assumption $v_{\mathrm{T}}<c$. Line 1-a in the figure shows how $\lambda_{m}$ varies due to the adiabatic 'cooling' of the turbulence alone. It is assumed here that the turbulence velocity has the Kolmogorov spectrum $v_{T}(\lambda) \propto \lambda^{1 / 3}$. The actual attenuation of the turbulence, taking account of the turbulence dissipation process, is presumably much more rapid than is indicated by line 1-a. Line 1-b is a free-hand estimate of what both processes together might do.

Lines 2 in the figure are estimates of the photon mean free path as a function of redshift. Line 2-a is computed on the assumption that the recombination of the plasma is unaffected by the turbulence. For line 2-b it is assumed that the matter remains fully ionized. 
One can imagine that there is fully developed turbulence on scales extending from line 1-b to somewhere above line 2-b. In this range, matter and radiation move roughly like a single fluid, the radiation providing the pressure and making the turbulence sub-sonic. Now the question is, what happens when lines 1 and 2 cross? We have the following possibilities :

(a) The plasma recombines rapidly, following line 2-a. Matter and radiation sharply decouple, leaving turbulent currents that are supersonic, and can slap together because $v_{\mathrm{T}}(\lambda) t / \lambda>1$. I presume the matter would then pile up in shocks, forming lumps much denser than the mean, and that these lumps would be gravitationally bound systems. This would not necessarily be a good thing, because the lumps would be much denser than galaxies, but conceivably the lumps could form the nuclei of galaxies, or quasars, or some sort of undiscovered object.

(b) Dissipation of the turbulence keeps the plasma ionized. Then the turbulence 'dies' at $z \gtrsim 300$, and the matter recombines. But this leaves residual matter currents with velocity $v_{\mathrm{T}}(\lambda) \sim \lambda / t$ at the point of intersection of the lines 1 and 2 , for the dissipation process ceases once the turbulence becomes weak. These residual currents can be treated by perturbation theory (Peebles, 1971b). One finds that the currents feed matter density irregularities, and that, if $v_{\mathrm{T}} \sim \lambda / t$ when the strong turbulence dies, gravitationally bound systems form shortly thereafter. Again, this is not necessarily a good thing, for the redshift is still high.

(c) The turbulence dies before the matter recombines. Then the radiation drag can sharply reduce the residual matter currents, avoiding the difficulty with premature formation of bound systems. Here the main point of interest is the residual acoustic 'noise' generated by the turbulence. This 'noise' may grow under the influence of gravity, fragmenting in the course of time into bound systems like galaxies. As was remarked, the attractive feature of this game is that the noise spectrum may be determined by the physics of fully developed turbulence. This is a subject that is just coming into lively discussion, and may well turn out to be profitable.*

In all the preceding discussion it has been imagined that the primeval turbulence can be treated as a perturbation to the Friedman-Lemaitre cosmological model $\left(v_{\mathrm{T}}<c\right)$. Rees (1972) has proposed dropping even this assumption, and introducing the picture of large initial fluctuations in space curvature on the scale of galaxies (although the Universe would have to be homogeneous and isotropic when averaged over scales $\sim 3000 h^{-1} \mathrm{Mpc}$, to account for the large-scale isotropy of the microwave background). A serious worry here, as in the milder versions of primeval turbulence, is that dense bound systems (including black holes) may form too soon in too great numbers.

One last aspect of primeval turbulence might be mentioned. It is often remarked that the rotation of the Galaxy might find a natural explanation as a residuum of primeval turbulence currents. ${ }^{* *}$ There is a simple problem with this idea if one assumes

\footnotetext{
* Acoustic noise generated by turbulence is discussed by Silk (1973), Jones (1973), and Stein (1974).
}

** cf. e.g. Silk (1973), Tomita (1972), Oort (1970). 
that the turbulence is left over from the radiation-dominated epoch. Let us concentrate attention on the 'main bulk' of our own Galaxy, the part within $10 \mathrm{kpc}$ radius of the centre. The mass contained within this radius is $\sim 10^{11} M_{\odot}$. If this mass were uniformly distributed within the sphere of $10 \mathrm{kpc}$ radius, the density would be $\sim 1$ proton $\mathrm{cm}^{-3}$. Now let us trace the expansion of the Universe back to the time when the mean cosmological density was equal to this mean value within the Galaxy, and let us suppose that, at this epoch, the material destined to end up in the Galaxy occupied a more or less spherical volume, radius $\sim 10 \mathrm{kpc}$. This happens at redshift $z \sim 50$ to 300 , depending on $\Omega$ and $h$. Finally, let us suppose that this roughly spherical proto-galaxy has angular momentum about its centre of mass equal to the angular momentum of the Galaxy. Since the Galaxy derives at least a significant fraction of its support from rotation, centrifugal force and self-gravitation are in a rough balance in the proto-galaxy at the epoch we are considering. Now what happens if we try to trace the evolution back still further in time? Can we assume that the protogalaxy remains as a coherent lump with its final angular momentum? It is hard to see how this could work, for compression ought to increase the ratio of centrifugal to self-gravitation forces, forcing apart the material. This would be a reasonable situation in fully developed turbulence, where there is a steady transfer of angular momentum among eddies. However, when our proto-galaxy reaches $10 \mathrm{kpc}$ radius we cannot invoke turbulence. The maximum optical depth of the proto-galaxy, if fully ionized, is 0.03 . Thus radiation can act only as a drag, not as a means of transferring angular momentum. Since the velocity of sound in the matter is much smaller than the assumed matter currents, $100 \mathrm{~km} \mathrm{~s}^{-1}$, collisions among eddies would be expected to produce shocks, not angular momentum transfer.

As is so often the case, this argument is hardly conclusive. It could be, for example, that primeval turbulence eddies are stabilized by some undiscovered process. The argument does show that there is considerable room for future work.

\subsection{Gravitational instability}

The gravitational instability picture also has been subject to doubt and uncertainty.* This effect figured in early discussions of structure in the big-bang cosmology, but then was criticized by later authors who felt that irregularities would grow too slowly to account for the origin of galaxies from 'reasonable' initial perturbations. The trend of some recent discussion has been in two directions - on the one hand, to reconsider what might be called a 'reasonable' initial density irregularity, and on the other to ignore the question of initial conditions as beyond our grasp for now, and to

* The role of gravitational instability in the evolution of structure in the expanding Universe was first considered by Lemaître (1933) and by Gamow and Teller (1939). For a review written at a time when the instability picture was less popular than it is now, see Layzer (1964). For reviews written after the renaissance, see the references in the first footnote of this report. 
concentrate instead on the 'uniformitarian' method.* The physics has not changed since the early criticisms, and the instability picture still is quite incapable of accounting for galaxies $a b$ initio. On the other hand, the uniformitarian method does lead to some limited but possibly significant results.

We have the picture that the matter within some $300 \mathrm{~h}^{-1} \mathrm{Mpc}$ (the depth of the Shane-Wirtanen survey) is distributed fairly smoothly overall and expanding in a roughly homogeneous and isotropic way according to Hubble's law, $v=H r$. Within this distance, matter is noticeably clumpy on a scale of $30 h^{-1} \mathrm{Mpc}$, but still expanding roughly in accordance with Hubble's law (for otherwise, peculiar velocities would be too high). The expected course of evolution of these $30 h^{-1} \mathrm{Mpc}$ irregularities is very simply described. Let $\varrho(\mathbf{r})$ be the mass density smoothed over a running average of scale $30 h^{-1} \mathrm{Mpc}$, and write

$$
\varrho(\mathbf{r})=\varrho_{0}(1+\delta(\mathbf{r})),
$$

where $\varrho_{0}$ is the mean and $\delta$ the fractional departure from the mean. Apparently $\delta \lesssim 1$. Then the method of linear perturbation theory says that if non-gravitational forces may be neglected the time variation of $\delta$ is a linear combination of growing and decaying modes. The decaying mode generally varies as $t^{-1}$. If the Universe is close to the Einstein-de Sitter model, where

$$
\varrho_{0} \cong \varrho_{\mathrm{c}}=3 H^{2} / 8 \pi \mathrm{G}
$$

so that $\Omega \cong 1$, and pressure may be neglected, then the growing mode varies as $t^{2 / 3}$. If $\Omega \ll 1$, the 'growing' mode is very nearly constant. But in this case, as we trace the expansion of the Universe back in time, we find that the value of $\Omega$ approaches unity (because $\varrho$ varies as $(1+z)^{3}, H^{2}$ as $(1+z)^{2}$ ). Since the local value of $\Omega$ is not less than $\sim 0.02$, we conclude that, unless some other effect intervenes, the growing mode would have been growing like $t^{2 / 3}$ at $z \gtrsim 50$.

These results do not depend very much on what the Universe is like at distances much greater than $300 h^{-1} \mathrm{Mpc}$. These distant parts can only affect the local situation through the intrusion of matter, which seems unlikely in view of the large peculiar velocities that would be required, or through the generation of tidal gravitational fields, which do not affect the time rate of change of $\delta$ in the linear approximation. Also, the very complicated and non-linear processes that obtain on scales much smaller than $30 h^{-1} \mathrm{Mpc}$ (rich clusters, galaxies) appear to be unimportant. The bulk motion of matter as measured by the coarse-grained average $\varrho(r)$ is not influenced by the complex details of the interaction of neighbouring galaxies, for these stresses cancel in pairs.

\footnotetext{
* The lack of enthusiasm for the instability picture mainly stems from the fact that the growing mode (or modes) grows as a power of time, not as an exponential. However, the argument that is occasionally advanced (cf. Layzer, 1964), that galaxies cannot grow out of 'reasonable' irregularities like thermal fluctuations, seems insecure because we have no theory to fix the time at which the initial fluctuations are set, and clearly the smaller this time the greater the growth factor. For discussion of irregularities in the early Universe, see Peebles (1968), Kundt (1971), Zel'dovich (1972), Harrison (1973). For discussion of 'uniformitarianism' in this context see Peebles $(1967,1972)$.
} 
Now let us consider the relative sizes of growing and decaying modes in $\delta$. We would be surprised to find that the decaying mode is very much larger than the growing mode, for that would require a cunning balance of irregularities in the matter and velocity fields. But we can imagine applying the same argument in the Universe as it was at a redshift $z \sim 10$, or 100 , or however large it might be without violating the basic picture and assumptions that have been evoked. Assuming this maximum redshift is large, we conclude that the decaying mode now must be much smaller than the growing mode.

How can one avoid this conclusion? The most direct way is to deny that the observed system of galaxies really is expanding, or, if expanding, has expanded from a dense initial state. In the model of Klein and Alfvén (cf. Alfvén, 1971), the observed system of galaxies is finite and has expanded from a point of maximum compression that perhaps is not very much denser than the present state. If during the course of expansion the effective value of $\Omega$ stays much less than unity, the conclusion does not apply. Perhaps the most difficult point for this picture is the isotropy of the microwave background. Since the system of galaxies would have an observable boundary, the microwave background would have to be an external sea of radiation through which most galaxies are moving at high speed, and it would be surprising that we do not observe this as a 24-h anistropy in the radiation intensity. Another possibility is to go to the Steady-State picture. Apparently the major problem here is the spectrum of the microwave background, which agrees with black-body in some detail (PC, pp. 129-142). In the framework of the big-bang cosmology, the argument is vitiated if non-gravitational phenomena are important. The difficulty of finding anything other than gravity that would materially affect the course of evolution of irregularities on a scale of $30 h^{-1} \mathrm{Mpc}$, at least back to $z \sim 1000$, helps explain why there has been so little controversy over the conclusion, but of course that is hardly a proof. The question is, to what extent can we account for the observed pattern of distribution and motion of matter as originating from small, growing irregularities in an expanding Universe? The rest of this section is devoted to a list of possible tests.

The available information on irregularities on a scale of $30 h^{-1} \mathrm{Mpc}$ is so scanty that it is hard to see how one could directly estimate the relative amplitude of growing and decaying modes. An interesting (and difficult) task will be to learn whether the details of the character of the large-scale distribution of galaxies, like the distribution function of the masses of systems of galaxies, could have evolved without contrivance from growing density irregularities. The work of de Vaucouleurs and Sandage and others to map out the local velocity field, within $\sim 30 h^{-1} \mathrm{Mpc}$, may be of decisive importance. For example, it might be hard to account for the rotation of the Local Supercluster, as proposed by de Vaucouleurs (cf. de Vaucouleurs and Peters, 1968) within the framework of the gravitational instability picture.

Under the instability picture, groups and clusters of galaxies fragment out as bound systems because the potential energy of interaction among separating mass elements exceeds the kinetic energy of expansion. One does not expect to find that associations of galaxies are freely expanding, with time-scales $\ll 10^{10} h^{-1} \mathrm{yr}$, unless there has been 
some violent mass loss. If the free expansion of groups and clusters can be truly established, it will have been demonstrated that the instability picture lacks some very essential element.

The rich compact clusters of galaxies appear to be simple systems, the major part of the mass being contained in a few hundred objects, the brightest galaxies. If these galaxies separated out as bound systems before the cluster formed, then the cluster formation process ought to be just as simple, and reasonably well approximated by a numerical N-body model.* It has been found that such models are quite successful in reproducing observed features of the Coma Cluster. Typically, the numerical computation commences when the proto-cluster is at the presumed point of maximum expansion, having just separated out from the general expansion as a distinct system. The $N$-body computation then follows the collapse and subsequent evolution. One finds that $10^{10} \mathrm{yr}$ is time enough for a proto-cluster to separate out from the general expansion, to collapse, and then relax to a state that matches the Coma Cluster fairly well - including the spatial distribution of galaxies and the variation of line-ofsight velocity dispersion with projected distance from the cluster centre. This is an important result because it shows that the instability picture can give a reasonable phenomenological account of at least some aspects of the large-scale structure. Of course one such example is hardly conclusive, because one certainly can think of other models for evolution of the clusters that could also fit the data.

In the gravitational instability picture in its most simple-minded form, a galaxy would originate as a collapsing cloud of gas (or, perhaps, of smaller clouds). This agrees with the picture Eggen et al. (1962) arrived at from a study of galactic structure, which may be significant, although we recognize that the connection is a tenuous one.

A simple property of galaxies that has been the subject of some controversy is their rotation. In the instability picture as outlined here, the initial growing density irregularities have negligible angular momentum and negligible circulation $(\nabla \times \mathbf{v} \cong 0)$. A proto-galaxy can pick up angular momentum, for it is an irregular lump in the tidal field of neighbouring developing proto-galaxies.** Two questions have been discussed:

(1) Does the proto-galaxy pick up enough angular momentum? Although there has been some discussion of this point, my impression is that the order of magnitude is about in the right range, considering the substantial uncertainties.

(2) What is the origin of the circulation of matter in galaxies? The matter is supposed to commence with negligible circulation, $\oint \mathbf{v} \cdot \mathrm{d} \mathbf{r} \cong 0$, for $\nabla \times \mathbf{v} \cong 0$. Under

\footnotetext{
* A first attempt at this was described by van Albada (1961). By using fast numerical computers I was able to make a more direct model computation, but still under the simplifying assumption that all galaxies have the same mass (Peebles 1970). The effect of a more realistic mass function has been discussed by Gunn and Aarseth (1972).

** This process was proposed by Hoyle (1949) and used by Sciama (1955) in his theory of the origin of galaxies in the Steady-State model. For a computation of the effect in the big-bang model, cf. Peebles (1969b). Oort (1970), Harrison (1971), Peebles (1971c). For the uncertainty in the angular momentum of the Galaxy, see Ostriker and Peebles (1973). For discussions of the circulation theorem, see Hunter (1970), Sunyaev and Zel'dovich (1972), Tomita (1973), Peebles (1973).
} 
the assumption that non-gravitational forces may be ignored (or more generally that the force per unit mass can be derived from a potential), Kelvin's circulation theorem says that $\nabla \times \mathbf{v}$ remains negligibly small. Does this contradict the observed circulation of matter in galaxies? It is my impression that here is a situation where the complexities of non-gravitational processes may quite becloud the theoretical position. Processes like turbulence, shocks, and fine-scale mixing all can violate the assumptions of the circulation theorem (cf. Peebles, 1973). As I understand the situation, therefore, the rotation of galaxies is not yet an embarrassment for the instability picture.

The available calculations of angular momentum transfer are at best estimates of 'typical' values. Before the angular momentum test can be tightened we will have to have not only more accurate calculations of the mean but also calculations of the expected dispersion about the mean. We might then hope to compare this dispersion with the frequency distribution of spirals and SO galaxies relative to ellipticals of diverse eccentricities. Another possible test is based on an expected scaling law: as long as the expansion of the Universe approximates to the Einstein-de Sitter model $(\Omega \sim 1)$, the mean square angular momentum transfer satisfies

$$
L^{2} E / G^{2} M^{5}=\text { constant, }
$$

where $M$ is the mass and $-E$ is the energy of the proto-system (kinetic plus potential, and neglecting dissipation). This says that, in the absence of dissipation, the eccentricity due to centrifugal flattening is independent of mass. The situation is complicated for proto-galaxies, because the collapse may be strongly dissipative, substantially increasing $E$. Proto-clusters of galaxies may provide a useful test, as these systems might be expected to conserve $E$. The Coma Cluster of galaxies shows no significant rotation, the limit being below the value expected from the scaling law. This is not yet a significant test, for we do not know the expected dispersion in the angular momentum, or the projection effects in the Coma Cluster. It will be interesting to have comparable redshift data on several more of the rich clusters. On the theoretical side, it will be interesting to study how the addition of angular momentum to the protocluster affects the evolution in the $N$-body models. Does a compact core still form? What is the rotation curve?

If galaxies formed before clusters, as seems reasonable (although certainly not demanded) in the instability picture, then how can one account for the special nature of the population of galaxies in rich clusters (more S0 galaxies, few spirals)? It still seems quite possible that some variant of the Baade-Spitzer collision process might account for superficial differences like gas content. It will be of considerable interest to learn whether the differences go deeper than that, whether the mass function of galaxies in clusters differs from the mass function for galaxies elsewhere, for example, or whether the angular momentum functions differ.

My final example is more indirect, involving even more assumptions, than the preceding ones. This is the proposal that globular star clusters might be the direct descendents of processes operating in the early Universe. One invokes here the bigbang cosmology and the Primeval Fireball. As has been remarked, in this cosmology 
at redshift $z \gtrsim 1000$ the Fireball radiation is hot enough to ionize the plasma, and Thomson scattering by the free electrons causes matter and radiation to act more or less like a single fluid. The pressure of the radiation is large, making the critical Jeans length for gravitational instability large, encompassing a mass comparable to that of clouds of galaxies. At $z \cong 1000$ the plasma is expected to recombine and decouple from the radiation. Now the critical Jeans length drops to a much smaller value. Under a fairly broad range of possible assumptions about the spectrum of density irregularities, the first systems to separate out from the general expansion would have mass fixed by this critical Jeans length.* The interesting coincidence is that this critical mass agrees with the typical masses of globular star clusters, systems which, by and large, are thought to be old, and which appear in what otherwise might be thought to be strange circumstances - around galaxies large and small, and even in intergalactic space. All this is highly suggestive, and I think therefore merits further careful attention. The picture must explain the differences among globular star clusters - the systematic variation of heavy element abundance with position in the Galaxy and the variations of globular star cluster abundances around otherwise similar-looking galaxies. At this point I have no idea whether these phenomena will prove embarrassing to the theory.

\section{Acknowledgement}

Research supported in part at Princeton by the National Science Foundation.

\section{References}

Abell, G. O.: 1958, Astrophys. J. Suppl. 3, 211.

Alfvén, H.: 1971, Phys. Today 24, 28.

Ambartsumian, V. A.: 1958, in R. Stoops (ed.), 11 th Solvay Conference on The Structure and Evolution of the Universe, Brussels, p. 241.

Ambartsumian, V. A.: 1965, in 13th Solvay Conference on The Structure and Evolution of Galaxies, Interscience Publishers, London. New York and Sydney, p. 1.

Arons, J.: 1972, Astrophys. J. 172, 553.

Arp, H.: 1971, Science 174, 1189.

Bahcall, J. N. and Joss, P. C.: 1972, Comments Astrophys. Space Phys. 4, 95.

Bogart, R. S. and Wagoner, R. V.: 1973, Astrophys. J. 181, 609.

Burbidge, G. R.: 1971, Nature 233, 36.

Burbidge, G. R., Burbidge, E. M., and Sandage, A.: 1963, Rev. Mod. Phys. 35, 947.

Dallaporta, N. and Lucchin, F.: 1972. Astron. Astrophys. 19, 123.

de Vaucouleurs, G.: 1971, Publ. Astron. Soc. Pacific 83, 113.

de Vaucouleurs, G. and Peters, W. L.: 1968, Nature 220, 868.

Doroshkevich, A. G., Zel'dovich, Ya. B., and Novikov, I. D.: 1967, Astron. Zh. 44, 295; Soviet Astron. 11, 233, 1967.

* For a discussion of the Jeans length in an expanding cosmological model see PC, pp. 217-220. Gamow first considered in detail the role of the Jeans length in cosmology (cf. Gamow and Teller, 1939). Recognizing that the Jeans length would be smaller than a typical large galaxy for the entropy per baryon that he and Alpher were contemplating (and that agrees well with modern observations), Gamow (1952) proposed that primeval turbulence might have increased the effective matter pressure, increasing the effective Jeans limit. Peebles and Dicke (1968) proposed that the naïve estimate of the Jeans mass be accepted, but interpreted as fixing globular cluster masses rather than galaxy masses. 
Eggen, O. J., Lynden-Bell, D., and Sandage, A.: 1962, Astrophys. J. 136, 748.

Field, G. B.: 1965, Astrophys. J. 142, 531.

Gamow, G.: 1952, Phys. Rev. 86, 251.

Gamow, G. and Teller, E.: 1939, Phys. Rev. 55, 654.

Gold, T. and Hoyle, F.: 1959, in R. N. Bracewell (ed.), 'Paris Symposium on Radio Astronomy', IAU Symp. 9, 583.

Gunn, J. E. and Aarseth, S. : 1972, preprint.

Harrison, E. R.: 1970a, Monthly Notices Roy. Astron. Soc. 147, 279.

Harrison, E. R.: 1970b, Monthly Notices Roy. Astron. Soc. 148, 119.

Harrison, E. R.: 1971, Monthly Notices Roy. Astron. Soc. 154, 167.

Harrison, E. R.: 1973, Ann. Rev. Astron. Astrophys. 11, 155.

Hauser, M. G. and Peebles, P. J. E.: 1973, Astrophys. J. 185, 757.

Holmberg, E.: 1969, Arkiv Astron. 5, 305.

Houck, J. R., Soifer, B. T., Harwit, M., and Pipher, J. L.: 1972, Astrophys. J. 178, L29.

Hoyle, F.: 1949, Problems of Cosmical Aerodynamics, Symp. of Int. Un. Theor. Appl. Math. and IAU, p. 195.

Hoyle, F.: 1958, in Proc. 11th Solvay Conf. on The Structure and Evolution of the Universe, p. 53.

Hoyle, F.: 1965, Galaxies, Nuclei and Quasars, Harper and Row, New York, pp. 18-20.

Hoyle, F. and Narlikar, J. V.: 1966, Proc. Roy. Soc. London A290, 143 and 185.

Hunter, C.: 1970, Astrophys. J. 162, 445.

Jeans, J.: 1928, Astronomy and Cosmogony, Cambridge University Press, p. 352.

Jones, B. J. T.: 1973, Astrophys. J. 181, 269.

Jones, B. J. T. and Peebles, P. J. E.: 1972, Comments Astrophys. Space Phys. 4, 121.

Kondo, M., Sofue, Y., and Unno, W.: 1971, Prog. Theor. Phys. Kyoto, Suppl. 49, 120.

Kundt, W.: 1971, Springer Tracts Mod. Phys. 58, 1.

Layzer, D.: 1964, Ann. Rev. Astron. Astrophys. 2, 341.

Lemaître, G.: 1933, Compt. Rend. Acad. Sci. Paris 196, 1085; Ann. Soc. Sci. Brussels A53, 51.

Limber, D. N.: 1954, Astrophys. J. 119, 655.

McCrea, W. H.: 1964, Monthly Notices Roy. Astron. Soc. 128, 335.

Milne, E. A.: 1948, Kinematic Relativity, Oxford, pp. 167-169.

Muehlner, D. J. and Weiss, R.: 1973, Phys. Rev. Letters 30, 757.

Ne'eman, Y.: 1965, Astrophys. J. 141, 1303.

Neyman, J., Scott, E. L., and Shane, C. D.: 1953, Astrophys. J. 117, 92.

Novikov, I. D.: 1964, Astron. Zh. 41, 1075; Soviet Astron. 8, 857, 1965.

Olsen, D. W. and Sachs, R. K.: 1973, Astrophys. J. 185, 91.

Oort, J. H.: 1958, in 11th Solvay Conference on The Structure and Evolution of the Universe, p. 163.

Oort, J. H.: 1970, Astron. Astrophys. 7, 381.

Ostriker, J. P. and Peebles, P. J. E.: 1973, Astrophys. J. 186, 467.

Ozernoy, L. M.: 1971, Astron. Zh. 48, 1160; Soviet Astron. 15, 923, 1972.

Ozernoy, L. M. and Chernin, A. D.: 1967, Astron. Zh. 44, 1131; Soviet Astron. 11, 907, 1968.

Peebles, P. J. E.: 1967, Astrophys. J. 147, 859.

Peebles, P. J. E. : 1968, Nature 220, 237.

Peebles, P. J. E.: 1969a, J. Roy. Astron. Soc. Can. 63, 27-28.

Peebles, P. J. E.: 1969b, Astrophys. J. 155, 393.

Peebles, P. J. E.: 1970, Astron. J. 75, 13.

Peebles, P. J. E.: 1971a, Physical Cosmology, Princeton University Press.

Peebles, P. J. E.: 1971b, Astrophys. Space Sci. 11, 443.

Peebles, P. J. E.: 1971c, Astron. Astrophys. 11, 377.

Peebles, P. J. E.: 1972, Comments Astrophys. Space Phys. 4, 53.

Peebles, P. J. E.: 1973, Publ. Astron. Soc. Japan 25, 291.

Peebles, P. J. E.: 1974, Astrophys. J. Suppl. No. 252.

Peebles, P. J. E. and Dicke, R. H.: 1968, Astrophys. J. 154, 891.

Peebles, P. J. E. and Hauser, M. G.: 1974, Astrophys. J. Suppl. No. 252.

Perko, T. E., Matzner, R. A., and Shepley, L. C.: 1972, Phys. Rev. D. 6, 969.

Rees, M. J.: 1971, in R. Sachs (ed.), Proc. Int. School of Phys. Enrico Fermi; Course XLVII, Academic

Press, New York and London, p. 315.

Rees, M. J.: 1972, Phys. Rev. Letters 28, 1669. 
Rees, M. J. and Reinhardt, M.: 1972, Astron. Astrophys. 19, 189.

Sciama, D. W.: 1955, Monthly Notices Roy. Astron. Soc. 115, 3.

Shane, C. D. and Wirtanen, C. A.: 1967, Publ. Lick Obs. 22, part 1.

Silk, J.: 1973, Comments Astrophys. Space Phys. 5, 9.

Silk, J. and Ames, S.: 1972, Astrophys. J. 178, 77.

Silk, J. and Lea, S.: 1973, Astrophys. J. 180, 669.

Silk, J. and Solinger, A. B.: 1973, Nature Phys. Sci. 244, 101.

Stecker, F. W. and Puget, J. L.: 1972, Astrophys. J. 178, 57.

Stein, R.: 1974, to be published.

Sunyaev, R. A. and Zel'dovich, Ya. B.: 1972, Astron. Astrophys. 20, 189.

Thorne, K. S.: 1967, Astrophys. J. 148, 51.

Tomita, K.: 1972, Prog. Theor. Phys. Kyoto 48, 1503.

Tomita, K.: 1973, Publ. Astron. Soc. Japan 25, 287.

Tomita, K., Nariai, H., Sato, H., Matsuda, T., and Takeda, H.: 1970, Prog. Theor. Phys. Kyoto 43, 1511.

van Albada, G. B.: 1961, Astron. J. 66, 590.

van der Kruit, P. C., Oort, J. H., and Mathewson, D. S.: 1972, Astron. Astrophys. $21,169$.

Weinberg, S.: 1972, Gravitation and Cosmology, Wiley, New York.

Williamson, K. D., Blair, A. G., Catlin, L. L., Hiebut, R. D., Loyd, E. G., and Romero, H. V.: 1973, Nature Phys. Sci. 241, 79.

Yu, J. T. and Peebles, P. J. E.: 1969, Astrophys. J. 158, 103.

Zel'dovich, Ya. B.: 1969, Astron. Zh. 46, 775; Soviet Astron. 13, 608, 1970.

Zel'dovich, Ya. B.: 1972, Monthly Notices Roy. Astron. Soc. 160, 1P.

Zel'dovich, Ya. B. and Novikov, I. D.: 1970, Astrofizica 6, 379.

\section{DISCUSSION}

Tifft: Why has rotation not been seen in clusters? Coma is well out of 'round' but shows no velocity gradient.

Peebles: In the gravitational instability picture, one could expect that clusters of galaxies may be out of round and have non-zero angular momentum. Because the two-body relaxation rate is slow, the oblateness need not be aligned along the angular momentum. It will be of considerable interest to learn whether the lack of a velocity gradient in the Coma cluster is found in other rich clusters.

G. de Vaucouleurs: Yes, it is true and surprising that no clear indication of general rotation has been detected in clusters or clouds of galaxies, even in ellipsoidal clouds of spirals, such as the S-cloud of the Virgo cluster (cf. Astrophys. J. Suppl. 5, 233, 1961).

Mestel: I am not clear as to where you agree and where you disagree with Lifshitz - is it that you assume large initial perturbations?

Peebles: The mathematics has not changed, only the interpretation. We observe that the Universe is now irregular. The perturbation analysis leads one to believe that the irregularities will increase with time and would have been less prominent in the past.

Mestel: Then perhaps you will be able to work backwards and learn something about the primeval turbulence?

Peebles: The great goal would be to determine whether the present nature of the matter distribution can be fitted to some combination of growing and decaying modes of irregularities, and, if so, to interpret the results in terms of the possible situation in the earlier Universe. Of course we are a long way from being able to do this.

Wright: Would you tell us how circulation might grow during the collapse of an originally noncirculating cloud of gas?

Peebles: Kelvin's law of conservation of circulation assumes ideal fluid flow with a single-valued relation between pressure and density. The collapse of a proto-galaxy seems likely to involve violent collisions among different parts, giving rise to turbulent dissipation and shock waves, both of which violate the assumptions of the circulation theorem. For details see the article in Publ. Astron. Soc. Japan 25, 291, 1973.

E. M. Burbidge: You said that the virial theorem takes hold in the condensations, and the film projected during your talk showed the persistence of these condensations. Does this mean we must find the 'missing mass' sufficient to balance the kinetic energy and stabilize the clusters? 
Peebles: Yes. If there is a large difference between the kinetic energy and the magnitude of the potential energy for typical groups or clusters, it will show that the gravitational instability picture is in serious trouble.

Ekers: In your statistical analysis showing the $30 \mathrm{Mpc}$ scale length, can you distinguish between superclusters of clusters and single clusters with a large halo of galaxies?

Peebles: I suspect both effects are involved. One observes that positions of cluster centres are correlated, and that there is a general halo of galaxies (or groups or small clusters of galaxies) around the average rich cluster. 